\title{
Lay Caregivers Education in Multicomponent Compression Bandaging in Obese Patients with Lower Limb Edema: A Case-Control Pilot Study
}

\author{
Tomasz Gradalski, MD, $\mathrm{PhD}^{1}$ and Katarzyna Ochalek, $\mathrm{PT}, \mathrm{PhD}^{1,2}$
}

\begin{abstract}
Background: A report on the usefulness of the instruction of lay carers in multilayer short-stretch compression bandaging within the initial intensive phase physical treatment of mixed-etiology chronic lower limb edema. Methods and Results: In a group of adult obese patients (including 24 females) with venous insufficiency and chronic bilateral lower limb edema, and without a history of physical therapy, 20 (ambulatory managed) were bandaged once daily (four layers, short stretch with cotton tube, and foam padding underneath) for 3 weeks (Monday-Friday) by skilled physiotherapists, and in 20 cases (education group, EG), the patients' lay carers were educated by these physiotherapists according to the same regime during one session. The outcome measures included limb volume (the method of circumference measurement with a tape at $4 \mathrm{~cm}$ intervals) after 1,3 , and 6 months, the time to reach the maintenance phase, the frequency of complementary bandaging during this phase, and the sense of self-efficacy (General Self-Efficacy scale, GSEs). No significant differences were noted between the two groups in the baseline measurements of age, body mass, and limb volume. The carers were able to apply compression bandages in all cases during the observation period. The time to reach the maintenance phase was longer in EG (6 vs. 1 weeks; $p<0.001)$. A similar median reduction in edema volume was observed at the end of the bandaging period, which continued for 3 and 6 months. It was only in EG that further improvement between 1 and 3 months was observed $(p=0.008)$. All participants represented an equally high optimistic sense of personal competence (GSEs).
\end{abstract}

Conclusions: The instruction of lay carers in bandaging may provide a simple clinically effective solution for lower limb edema management, thus lowering its costs.

Keywords: lymphedema, venous insufficiency, compression bandage, caregivers, lower limb

\section{Introduction}

L OWER LIMB EDEMA of mixed etiology (phlebolymphe1 dema) is the most common cause of swelling in the Western world. ${ }^{1}$ Diuretics, a frequent therapeutic option in such cases, may induce chronicity of the edema in the long term due to disturbances of the renin-angiotensin and antidiuretic hormone relationship. ${ }^{2}$ Compression therapy, recommended to decrease venous and lymphatic hypertension, is widely used in the treatment of mixed edema, skin changes, and ulcerations. ${ }^{3}$ Self-performance of complex decongestive therapy, based on compression, manual lymphatic drainage, and remedial exercises, can be a valuable method to decrease limb volume and chronic pain in breast cancer-related lymphedema. ${ }^{4,5}$ Self-bandaging programs for lymphedema patients have been promoted in many countries, for example, in Canada, ${ }^{6}$ Ireland, or the Netherlands. ${ }^{7}$ Although many lifestyle changes and self-care indications for patients with lower limb edema are proposed, ${ }^{8}$ the utility of self-applied short-stretch leg compression has not been evaluated, except for adjustable wrap compression systems. ${ }^{9}$ The objective of this retrospective casecontrol pilot study was to report on the usefulness of the instruction of lay carers in multilayer short-stretch compression bandaging within the initial intensive phase physical treatment of mixed-etiology chronic lower limb edema. The rationale for this is to enable the effectiveness of this cost-cutting management.

${ }^{1}$ St Lazarus Hospice, Krakow, Poland.

${ }^{2}$ Department of Clinical Rehabilitation, Faculty of Motor Rehabilitation, University of Physical Education, Krakow, Poland. 


\section{Materials and Methods}

Forty adult obese patients (including 24 females) with venous insufficiency (all stage Cs4a-Es according to CEAP classification) and chronic bilateral lower limb edema without a history of physical therapy were selected. Twenty (ambulatory managed - control group, AM) were bandaged (8 and $10 \mathrm{~cm}$ width, Rosidal K, Lohmann and Rauscher, Germany-four layers, short stretch with cotton tube, and foam padding underneath) by skilled physiotherapists once daily from Monday to Friday (bandages applied were left on day and night to the next session, also through the weekends). In 20 cases (education group, EG), the patients' lay carers were educated by these physiotherapists according to the same regime during one session. Both groups were encouraged to maintain normal activity including walking with bandages applied.

After 1 week in AM and 3 weeks in EG, the patients were checked for the pitting signs of edema. When it persisted, further bandaging was advised for 1 week. When pitting edema was absent, the patients were fitted with made-tomeasure flat-knitted thick and stiff compression stockings (MEDI Bayreuth, Germany-CCL class 2; 23-32 mmHg) and advised to wear them on a daily basis with sustained activity (maintenance phase). Lifestyle changes comprising aerobic physical activity and lowering of calorie intake, aiming at losing weight, were recommended in both groups.

Limb volume (circumference measured with a tape at $4 \mathrm{~cm}$ intervals, from the ankle-knuckle up to the knee, with simplified frustrum volume formula method) was measured at the end of the intensive management phase (after 1 week in $\mathrm{AM}$ and 3 weeks in EG), then 3 and 6 months later. Both absolute (in $\mathrm{mL}$ ) volume and a weight-adjusted volume change (WAC) ${ }^{10}$ after 6 months were used to assess the results of physiotherapy. The time to reach the maintenance phase and the frequency of complementary bandaging needed during this phase were monitored. The General SelfEfficacy scale (GSE), ${ }^{11}$ which is a measure of a sense of personal competence to deal effectively with stressful situations and has been a routine procedure at the clinic, was additionally analyzed.

The normality of data distribution was analyzed with the Shapiro-Wilk test. The Wilcoxon signed-rank test was used to compare ordinal data during each time period. In terms of the comparison between the subgroups of patients, the Mann-Whitney test was employed. Spearman's rank correlation was used to assess the relationship between the volume changes and GSE.

The research was conducted in accordance with the Helsinki Declaration. Informed consent from patients was not required due to the retrospective character of the study. Institutional review board approval for this retrospective study was obtained.

\section{Results}

No significant differences were noted between the two groups in the baseline measurements of age, body weight, body mass index (BMI), and edema duration (Table 1). Stemmer sign was present in 10 patients in each group. Both groups represented an equally high optimistic sense of personal competence (GSEs).
Table 1. Characteristics of the Two Groups OF PATIENTS

\begin{tabular}{|c|c|c|c|c|c|}
\hline \multirow[b]{2}{*}{ Parameter } & \multicolumn{2}{|c|}{$\begin{array}{l}\text { Education } \\
\text { group }\end{array}$} & \multicolumn{2}{|c|}{$\begin{array}{c}\text { Ambulatory } \\
\text { group }\end{array}$} & \multirow[b]{2}{*}{$\mathrm{p}$} \\
\hline & Median & $I Q R$ & Median & $I Q R$ & \\
\hline Age (years) & 65.0 & 19.0 & 64.0 & 13.0 & 0.7 \\
\hline Body weight (kg) & 110.5 & 23.8 & 112.5 & 13.0 & 0.8 \\
\hline Height $(\mathrm{m})$ & 1.6 & 0.1 & 1.7 & 0.1 & 0.7 \\
\hline BMI $\left(\mathrm{kg} / \mathrm{m}^{2}\right)$ & 39.5 & 6.8 & 39.8 & 8.6 & 0.9 \\
\hline $\begin{array}{l}\text { Edema duration } \\
\text { (months) }\end{array}$ & 96.0 & 112.5 & 120.0 & 125.0 & 0.8 \\
\hline GSEs (points) & 32.0 & 5.5 & 33.0 & 5.5 & 0.8 \\
\hline $\begin{array}{l}\text { Bandaging therapy } \\
\text { (weeks) }\end{array}$ & 6.0 & 1.0 & 1.0 & 0.0 & $<0.001$ \\
\hline
\end{tabular}

BMI, body mass index; GSE, General Self-Efficacy scale; IQR, interquartile range; $p$, Mann-Whitney $U$ test.

In $\mathrm{EG}$, the mean weight increased by $3.3 \mathrm{~kg}$, whereas in AM, it decreased by $2.0 \mathrm{~kg}$, but the BMI did not change significantly within 6 months of observation in each group $(p=0.13)$. The carers were able to apply compression bandages in all cases for the observed period. The median time to achieve the maintenance phase in EG was longer (6.0 vs. 1.0 weeks; $p<0.001)$. Thirteen patients in both groups occasionally required additional compression bandaging during the maintenance phase. Within EG, no additional ambulatory visits were necessary, whereas in AM, one or two interventional visits per patient were appointed.

A significant improvement was observed in both groups during the intensive phase. In the first 3 months of maintenance phase, further improvement was seen in EG only (Fig. 1). In both groups, the reduction of limb volumes was maintained within 6 months of observation.

Group comparison revealed a similar mean reduction in edema, expressed in both absolute (mL) and relative (WAC) volumes (Table 2). No particular correlations were seen between GSE and limb volume changes.

\section{Discussion}

The presented data show that ambitious lay caregivers having a high optimistic sense of personal competence can be effectively taught how to apply multicomponent compression bandaging to edematous legs, and that the intensive phase of edema physiotherapy based on carers' activity may be sufficiently effective in longer observation.

"Inelastic" - thick and stiff compression, more prominent in standing position and on walking (with higher working pressures and lower resting pressures), making it more comfortable to wear-is recommended for several serious clinical conditions (e.g., skin ulcerations and various types of edema). Both bandages and stockings perform on the same principle: the higher the stiffness, the larger the improvement of hemodynamic parameters and the more effective the reduction of edema. ${ }^{12}$ As most cases of chronic lower limb edema are of compound origin, affecting eventually the lymphatic output, principles of conservative management of lymphatic congestion may be extrapolated into the cases of chronic edema and incorporated into the community health system. ${ }^{13}$ Mixedetiology limb edema can be one of the major complaints not 


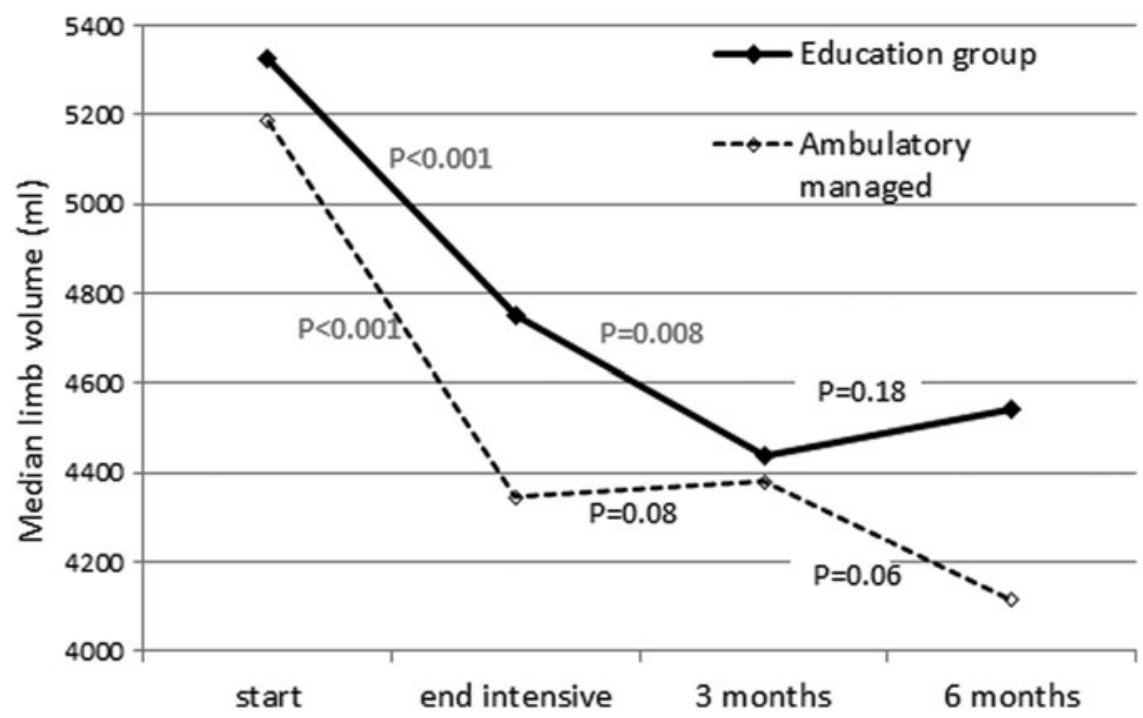

FIG. 1. Median limb volumes (mL) during the study in EG and AM groups. The median time of intensive phase in EG and AM groups was 6 and 1 week, respectively. ( $p$ value Wilcoxon signed-rank test). AM, ambulatory managed; EG, education group.

only among the elderly population, ${ }^{14}$ but also within hospice patients. ${ }^{15}$ Our observation suggests the utility of implementation of caregiver-based compression therapy in these numerous cases, also within the end-of-life population.

One of the most important prerequisites for efficacious bandaging is to apply them not too loosely and with a proper frequency, and the main problem concerning compression therapy is the lack of adequately trained staff. ${ }^{16}$ Compression wraps, commonly advised lately in cases of venous insufficiency, despite obtaining less pressure range variation, however, also need detailed instruction at the demonstration course, ${ }^{9}$ and they can be even eight times more expensive than the multicomponent set of short-stretch bandaging products comprising cotton stockinettes, foam paddings, and bandages (in Poland the price difference is 130 USD vs. 16 USD, and neither cost is reimbursable under the national insurance scheme). Self-adjustable (sometimes more often than once a day) compression devices were more effective than mere daily bandaging in reducing venous leg edema within the first week of initial therapy owing to higher and more stable pressures obtained during the whole day. ${ }^{17}$ Therapy initiation with a single (with moderate pressure of $23-32 \mathrm{mmHg})^{18}$ or two superimposed elastic stockings, ${ }^{19}$ which could be as efficient as inelastic bandages, was not considered in our patients due to financial constrains. In our observation, ambulatory intensive phase of therapy with bandaging was broadly acceptable, typically also lasted 1 week, but it might be necessary for a longer period when compression is applied by a caregiver.

Clinicians play a virtual role in supporting patients to know about, perform, and believe in the importance of selfmanagement strategies for maintaining the achieved results in long follow-up. ${ }^{20}$ The majority $(81 \%)$ of patients can apply ordinary elastic bandages for venous leg ulcer management by themselves, ${ }^{21}$ but the self-application of the short-stretch bandages is more problematic, as the variation in the strength of application generates higher fluctuations in obtained compression under the bandage. In addition, self-bandaging of lower legs by older obese subjects, frequently with comorbidities, may not be possible in the majority of cases. ${ }^{9}$ The quality of self-applied compression bandages in patients with chronic venous insufficiency may often be deficient: in one study, nearly a half of them could not afford it due to physical limitations and only one-fifth of those who could apply bandages achieved effective compression pressure. ${ }^{22}$

Table 2. Characteristics of Two Managed Groups

\begin{tabular}{|c|c|c|c|c|c|}
\hline \multirow[b]{2}{*}{ Limb volume $(m L)$} & \multicolumn{2}{|c|}{ Educational group } & \multicolumn{2}{|c|}{ Ambulatory group } & \multirow[b]{2}{*}{$\mathrm{p}$} \\
\hline & Median & $I Q R$ & Median & $I Q R$ & \\
\hline Initially & 5327.0 & 1299.5 & 5185.0 & 1276.3 & 0.8 \\
\hline After intensive phase bandaging & 4749.0 & 1205.0 & 4343.5 & 1170.5 & 0.5 \\
\hline Change within intensive phase & -457.5 & 856.0 & -730.5 & 695.0 & 0.057 \\
\hline After 3 months of maintenance therapy & 4437.0 & 1196.5 & 4378.5 & 1184.3 & 0.8 \\
\hline Change within 3 months & -733.5 & 933.0 & -724.5 & 866.8 & 0.6 \\
\hline After 6 months of maintenance therapy & 4542.0 & 1215.5 & 4113.0 & 1207.3 & 0.7 \\
\hline Change within 6 months & -757.5 & 995.8 & -777.5 & 991.0 & 0.3 \\
\hline WAC within 6 months $(\%)$ & -14.6 & 12.6 & -14.1 & 15.4 & 0.8 \\
\hline
\end{tabular}

WAC, weight-adjusted volume change. 
Surprisingly, even experienced nurses and therapists usually miss the target range of compression pressures. ${ }^{23}$ However, in lymphedema patients, also with lower limbs affected, successful attempts at the self-application of multicomponent short-stretch bandages have already been reported. ${ }^{6}$ In these patients the effectiveness of such bandaging was comparable with a precast adjustable compression system in terms of excess limb volume reduction and symptoms control. ${ }^{24}$

The existing self-bandaging programs assume the participation of caregivers routinely or in cases when the patient cannot cooperate sufficiently. ${ }^{6}$ The main rehabilitation strategies for poorly active and obese patients encompass supervision at home in many activities, including compression. ${ }^{8}$ In low-income areas of endemic filarial infections, this strategy, based on proper skin care and bandaging training (also for carers) at day centers, is being developed as a part of the World Health Organization guidelines. ${ }^{25}$ Our study also supports the utility of such caregivers' aid.

Mixed lower limb edema as a chronic condition with a high risk of exacerbations needs an ongoing systematical therapeutic regime. In exacerbations, an additional bandaging is often necessary to reduce the edema. ${ }^{26}$ Besides high selfefficacy that may enhance self-care, ${ }^{27}$ knowledge of the bandaging technique is necessary to maintain a therapy that is adjusted to the actual condition. The second prerequisite also encourages better education of patients and their caregivers.

There are some limitations to this study. First, we have not monitored the compression pressures generated under the bandages due to retrospective design. Some observations indicate that self-application of compression wraps may produce more consistent pressures through the day than mutual bandaging, but concurrently the patient's subjective feeling of strong yet not painful compression can be a sufficient reliable guide during both applications. ${ }^{9}$ Second, we have performed circumference measurements only at the level of the lower legs, where compression was applied. Third, the small sample of solely obese participants and the retrospective design of this study imply the possibility of recall bias.

\section{Conclusions}

Earlier results demonstrate that the instruction of lay caregivers in bandaging may provide a simple and clinically effective solution for lower limb edema management. However, the time to the stable phase permitting for a shift from bandaging to the wearing of compression hosiery is longer when lay carers are performing physiotherapy. The follow-up results indicate that both methods could similarly improve lower limb edema. A larger prospective study is warranted.

\section{Authors Disclosure Statement}

No competing financial interests exist.

\section{Funding Information}

No funding was received for this study.

\section{References}

1. Farrow W. Phlebolymphedema-A common underdiagnosed and undertreated problem in the wound care clinic. J Am Col Certif Wound Spec 2010; 2:14-23.
2. Bartoli E, Rossi L, Sola D, Castello L, Sainaghi PP, Smirne C. Use, misuse and abuse of diuretics. Eur J Intern Med 2016; 39:9-17.

3. Gloviczki P, Comerota AJ, Dalsing MC, et al. The care of patients with varicose veins and associated chronic venous diseases: Clinical practice guidelines of the Society for Vascular Surgery and the American Venous Forum. J Vasc Surg 2011; 53(5 Suppl.):2S-48S.

4. Ligabue MB, Campanini I, Veroni P, Cepelli A, Lusuardi M, Merlo A. Efficacy of self-administered complex decongestive therapy on breast cancer-related lymphedema: A single-blind randomized controlled trial. Breast Cancer Res Treat 2019; 175:191-201.

5. Shallwani SM, Towers A. Self-management strategies for malignant lymphedema: A case report with 1-year and 4year follow-up data. Physiother Canada 2018; 70:204-211.

6. Tidhar D, Hodgson P, Shay C, Towers A. A lymphedema self-management programme: Report on 30 cases. Physiother Canada 2014; 66:404-412.

7. Damstra RJ. Self-management in lymphedema. Veins Lymphat 2018; 7:7987.

8. Caggiati A, De Maeseneer M, Cavezzi A, Mosti G, Morrison N. Rehabilitation of patients with venous diseases of the lower limbs: State of the art. Phlebology 2018; 33:663671.

9. Partsch H. Reliable self-application of short stretch leg compression: Pressure measurements under self-applied, adjustable compression wraps. Phlebology 2019; 34:208213.

10. Miller CL, Specht MC, Horick N, et al. A novel, validated method to quantify breast cancer-related lymphedema (BCRL) following bilateral breast surgery. Lymphology 2013; 46:64-74.

11. Scholz U, Gutierrez-Dona B, Sud S, Schwarzer R. Is perceived efficay a universal construct? Psychometric findings from 25 countries. Eur J Psychol Assess 2002; 18:242-251.

12. Bjork R, Ehmann S. S.T.R.I.D.E. Professional guide to compression garment selection for the lower extremity. J Wound Care 2019; 28(6 Suppl. 1) 1-44.

13. Moffatt CJ, Doherty DC, Franks PJ, Mortimer PS. Community-based treatment for chronic edema: An effective service model. Lymphat Res Biol 2018; 16:92-99.

14. Thaler HW, Wirnsberger G, Pienaar S, Roller RE. Bilateral leg edema in the elderly. Clinical considerations and treatment options. Eur Geriatr Med 2010; 1:353-357.

15. Gradalski T. Edema of advanced cancer: Prevalence, etiology, and conservative management-A single hospice cross-sectional study. J Pain Symptom Manage 2019; 57: 311-318.

16. Partsch H. Compression heals leg ulcers due to abolishment of venous reflux. J Wound Care 2019; 28:427.

17. Mosti G, Cavezzi A, Partsch H, Urso S, Campana F. Adjustable Velcro ${ }^{\circledR}$ compression devices are more effective than inelastic bandages in reducing venous edema in the initial treatment phase: A randomized controlled trial. Eur J Vasc Endovasc Surg 2015; 50:368-374.

18. Mosti G, Picerni P, Partsch H. Compression stockings with moderate pressure are able to reduce chronic leg oedema. Phlebology 2012; 27:289-296.

19. Mosti G, Partsch H. Bandages or double stockings for the initial therapy of venous oedema? A randomized, controlled pilot study. Eur J Vasc Endovasc Surg 2013; 46: 142-148. 
20. Kapp S, Miller C. The experience of self-management following venous leg ulcer healing. J Clin Nurs 2015; 24: 1300-1309.

21. Suehiro K, Morikage N, Harada T, Samura M, Takeuchi Y, Mizoguchi T, Hamono K. Self-care-based treatment using ordinary elastic bandages for venous leg ulcers. Ann Vasc Dis $2017 ; 10: 229-233$.

22. Stoffels-Weindorf M, Stoffels I, Jockenhöfer F, Dissemond J. Quality of self-applied compression bandages in patients with chronic venous ulcers: Results of a prospective clinical study. Hautarzt 2018; 69:306-312.

23. Heyer K, Protz K, Augustin M. Compression therapyCross-sectional observational survey about knowledge and practical treatment of specialised and non-specialised nurses and therapists. Int Wound J 2017; 14:1148-1153.

24. Pujol-Blaya V, Salinas-Huertas S, Catasús ML, Pascual T, Belmonte R. Effectiveness of a precast adjustable compression system compared to multilayered compression bandages in the treatment of breast cancer-related lymphoedema: A randomized, single-blind clinical trial. Clin Rehabil 2019; 33:631-641.
25. World Health Organization. Lymphoedema Staff Manual. Geneva: WHO; 2001.

26. Badger CM, Peacock JL, Mortimer PS. A randomized, controlled, parallel-group clinical trial comparing multilayer bandaging followed by hosiery versus hosiery alone in the treatment of patients with lymphedema of the limb. Cancer 2000; 88:2832-2837.

27. Fukuda R, Akazawa C, Arakawa C. Relationship between self-care continuation and self-efficacy in patients with secondary lower extremity lymphedema: A questionnaire survey 2015; 5:57-62.

\author{
Address correspondence to: \\ Tomasz Gradalski, MD, PhD \\ St Lazarus Hospice \\ Fatimska 17 \\ Krakow 31-831 \\ Poland
}

E-mail: tomgr@mp.pl 